\title{
Correction to: Applying Facial Expression of Augmented Reality for Teaching Students with Autism Spectrum Disorders
}

Lai-Chung Lee and Kuo-Chen Kao

\begin{abstract}
Correction to:
Chapter "Applying Facial Expression of Augmented Reality for Teaching Students with Autism Spectrum Disorders" in: T. Ahram and C. Falcão (Eds.): Advances in Usability, User Experience, Wearable and Assistive Technology, AISC 1217, https://doi.org/10.1007/978-3-030-51828-8_112
\end{abstract}

In the original version of the book, the following belated corrections have been incorporated:

In chapter "Applying Facial Expression of Augmented Reality for Teaching Students with Autism Spectrum Disorders", the affiliation "Department of Designing National Taipei University of Technology Taipei Taiwan" of author "Lai-Chung Lee" has been changed to "Department of Interaction Design, National Taipei University of Technology, Taipei, Taiwan", and the affiliation "Department of Designing National Taipei University of Technology Taipei Taiwan" of author "Kuo-Chen Kao" has been changed to "College of Design, National Taipei University of Technology, Taipei, Taiwan”. 